Mourad TAFER ${ }^{1,2}$

Said SLIMANI ${ }^{3}$

Dalila KHERCHOUCHE ${ }^{4}$

Farid BEKDOUCHE ${ }^{5,6}$

Mahand MESSAOUDÈnE ${ }^{7 *}$

1 Université de Bejaia

Faculté des sciences de la Nature et de la Vie 06000 Bejaia

Algérie

${ }^{2}$ Université de Bouira

Faculté des sciences de la Nature et de la Vie et des sciences de la Terre

Laboratoire de gestion et valorisation des ressources naturelles et assurance qualité

Rue Drissi Yahia

10000 Bouira

Algérie

${ }^{3}$ Université de Tizi-Ouzou

Faculté des sciences biologiques

et agronomiques

15000 Tizi-Ouzou

Algérie

\begin{abstract}
${ }^{4}$ Université Batna 1
Institut des sciences vétérinaires

et des sciences agronomiques

Allées 19 mai, route de Biskra

05000 Batna

Algérie
\end{abstract}

${ }^{5}$ Université Batna 2

Faculté des sciences de la Nature et de la Vie 53 , route de Constantine

Fésdis, 05078 Batna

Algérie

${ }^{6}$ Université de Bejaia

Laboratoire de recherche en écologie et environnement

06000 Bejaia

Algérie

${ }^{7}$ Institut national de recherche forestière (INRF) Station régionale de Tizi-Ouzou

BP 30

15365 Yakouren

Algérie

* Directeur de recherche à l'Institut national de recherche forestière, station d'Azazga (Algérie), décédé le 18 novembre 2016.

Auteur correspondant /

Corresponding author:

Mourad Tafer-m.tafer@univ-bouira.dz

\section{Événements climatiques extrêmes et croissance radiale de Cedrus atlantica (Manetti) dans les massifs du Belezma et du Chélia (Algérie)}

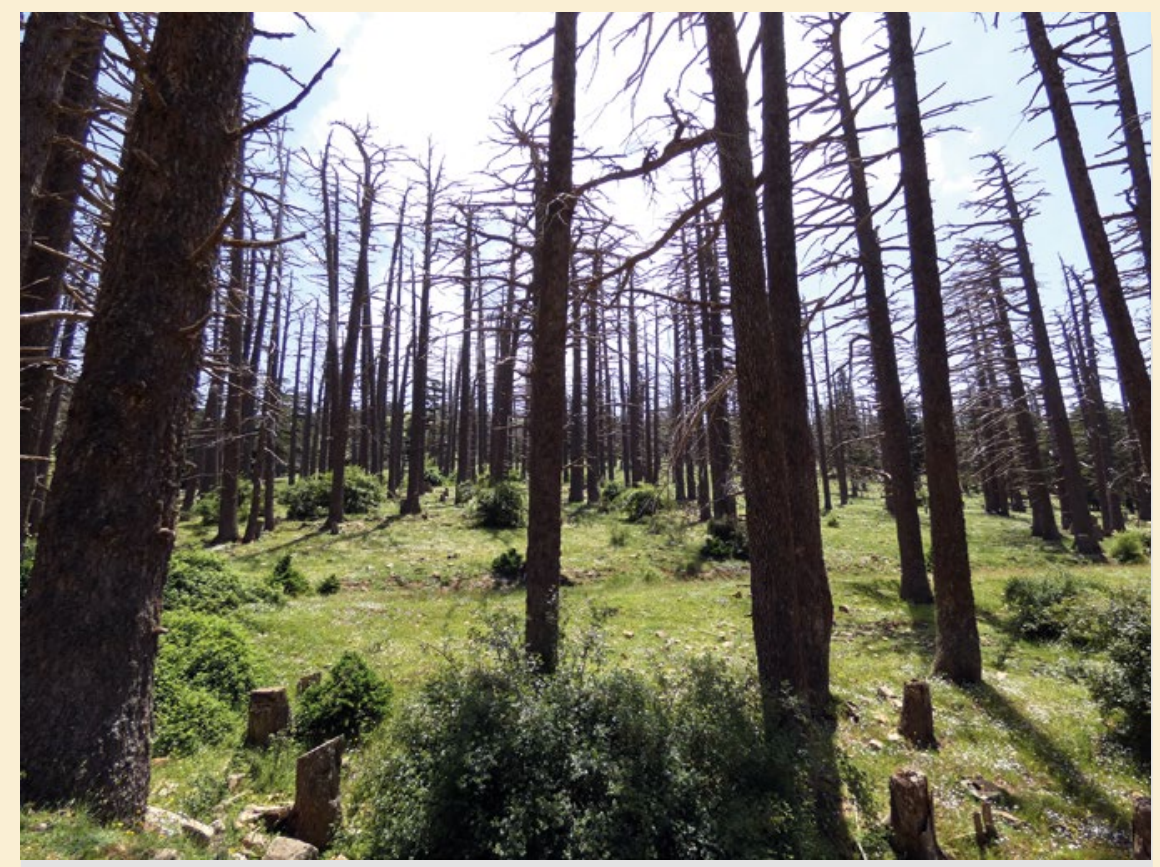

Photo 1.

Ampleur du dépérissement dans la cédraie du Belezma.

Extent of dieback in the Belezma cedar forest.

Photo Parc national du Belezma.

Doi : 10.19182/bft2021.350.a36298 - Droit d'auteur (c) 2021, Bois et Forêts des Tropiques - (c) Cirad - Date de soumission : 13 janvier 2021 ;

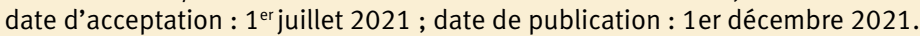
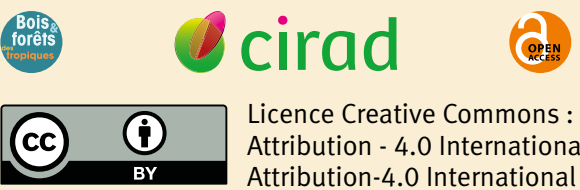

Licence Creative Commons :

Attribution - 4.0 International.

Attribution-4.0 International (CC BY 4.0)
Citer l'article / To cite the article

Tafer M., Slimani S., Kherchouche D., Bekdouche F., Messaoudène M., 2021. Événements climatiques extrêmes et croissance radiale de Cedrus atlantica (Manetti) dans les massifs du Belezma et du Chélia (Algérie). Bois et Forêts des Tropiques, 350 : 43-56. Doi : https://doi.org/10.19182/bft2021.350.a36298 


\section{RÉSUMÉ}

\section{Événements climatiques extrêmes et croissance radiale de Cedrus atlantica (Manetti) dans les massifs du Belezma et du Chélia (Algérie)}

Pour mieux comprendre l'impact des facteurs climatiques et l'influence de la diversité des habitats sur le dépérissement des cédraies des Aurès, une approche dendrochronologique basée sur la relation cerne-climat a été adoptée et appliquée à six stations, réparties sur deux cédraies méridionales, du Belezma et du Chélia (Atlas saharien), distantes de $60 \mathrm{~km}$ et impactées par différentes perturbations climatiques. Pour les deux sites étudiés, un sondage de 120 arbres a été réalisé, permettant d'extraire au total 240 carottes. Une chronologie maîtresse a été établie pour chaque station et une série moyenne de données a été élaborée pour chaque site. Les chronologies obtenues ont été standardisées afin d'atténuer les effets et les tendances non liées à la variation climatique. Puis, les chronologies résiduelles ont été utilisées pour appréhender la relation cerne-climat. Les résultats obtenus montrent une hétérogénéité de la réponse du cèdre de l'Atlas au sein des deux massifs. La durée et l'intensité des épisodes secs se sont différemment exprimées en fonction des deux sites d'étude, avec un nombre d'années sèches plus important pour le massif du Belezma, pour lequel l'indice standardisé de précipitations (SPI) révèle une récurrence d'épisodes pluvieux plus sévères à partir de la seconde moitié du XXe siècle. Un taux élevé d'années caractéristiques a été enregistré au niveau des deux stations étudiées. Cependant, un plus grand nombre d'événements extrêmes est enregistré au Belezma en comparaison avec le Chélia. Le signal climatique revêt une bonne homogénéité dans les deux sites, mais il est mieux perçu au Belezma. Les coefficients de corrélation expriment une plus forte relation des précipitations avec la croissance radiale au sein de la cédraie dépérie du Belezma, où le dépérissement semble être aggravé par l'âge vieillissant de cette formation, sa basse altitude, son sol maigre et ses peuplements équiennes. À contrario, l'altitude élevée, le sol profond et la structure en futaie jardinée de la formation du mont Chélia sont des facteurs qui semblent atténuer cette forte corrélation cerne-climat, et par conséquent les effets du déficit hydrique responsables du dépérissement sont significativement réduits.

\section{ABSTRACT}

\author{
Extreme climatic events and radial \\ growth of Cedrus atlantica (Manetti) \\ in the Belezma and Chélia cedar forests \\ (Algeria)
}

To better understand the impact of climatic factors and the influence of habitat diversity on the decline of cedar forests in the Aurès range, a dendrochronological approach based on the relationship between tree rings and climate was adopted and applied in six stations located $60 \mathrm{~km}$ apart in two southern Algerian cedar forests, Belezma and Chélia (Saharan Atlas), which are affected by different climatic disturbances. A survey of 120 trees was carried out across the two sites studied, with a total of 240 core samples extracted. A baseline chronology was established for each station and an average data set developed for each site. The resulting chronologies were standardised in order to smooth out effects and trends not related to climatic variations. The residual chronologies were then used to understand the relationship between tree rings and climate. The results show an uneven response of Atlas cedars in both forests. The duration and intensity of dry episodes were expressed differently in the two study sites, with a larger number of dry years for the Belezma forest, for which the standardised precipitation index (SPI) reveals a recurrence of more severe rainy episodes from the second half of the 20th century. A high rate of characteristic years was recorded at the two stations studied. However, there were more extreme events recorded at Belezma than Chélia. The climatic signal is homogeneous in both sites but more clearly perceived in Belezma. The correlation coefficients show a stronger relationship between precipitation and radial growth in the declining cedar forest of Belezma, where dieback seems to be worsened by the age of the stand and its low altitude, poor soil and even-aged structure. On the other hand, the high altitude, deep soil and high forest structure of the Chélia forest are factors that seem to mitigate the strong correlation between tree-rings and climate, so that the effects of the water deficit responsible for dieback are significantly reduced.

Keywords: Cedrus atlantica, Atlas cedar, dieback, tree-ring relationship with climate, Aurès, Algeria.

\section{RESUMEN}

\author{
Eventos climáticos extremos \\ y crecimiento radial del Cedrus atlantica \\ (Manetti) en los macizos de Belezma \\ y Chélia (Argelia)
}

Para comprender mejor el impacto de los factores climáticos y la influencia de la diversidad de hábitats en el declive de los bosques de cedros en Aurès, se adoptó un enfoque dendrocronológico basado en la relación de los anillos de crecimiento con el clima. Se aplicó a seis estaciones situadas en dos bosques de cedros del sur, Belezma y Chélia (Atlas sahariano), separados por $60 \mathrm{~km}$ y afectados por diferentes perturbaciones climáticas. En las dos zonas estudiadas, se realizó un estudio de 120 árboles, lo que permitió extraer un total de 240 muestras. Se estableció una cronología maestra para cada estación y se elaboró una serie de datos promedio para cada sitio. Las cronologías resultantes se normalizaron para suavizar los efectos y las tendencias no relacionados con la variación climática. A continuación, se utilizaron las cronologías residuales para comprender la relación anillo-clima. Los resultados obtenidos muestran una heterogeneidad de la respuesta del cedro del Atlas en los dos macizos. La duración y la intensidad de los episodios secos fueron diferentes en los dos bosques estudiados, con un mayor número de años secos para el macizo de Belezma, donde el índice de precipitación estandarizado (SPI) revela una recurrencia de episodios lluviosos más severos a partir de la segunda mitad del siglo XX. En las dos estaciones estudiadas se registró un alto índice de años característicos. Sin embargo, en Belezma se registra un mayor número de eventos extremos en comparación con Chelia. La señal climática es bastante homogénea en ambos sitios, pero se percibe mejor en Belezma. Los coeficientes de correlación muestran una relación más fuerte entre las precipitaciones y el crecimiento radial en el cedral debilitado de Belezma, donde la mortandad parece estar agravada por el envejecimiento del bosque, la baja altitud, el poco espesor del suelo y las masas forestales de edad homogénea. Por otro lado, la elevada altitud, la profundidad del suelo y la estructura de entresaca de la formación del Monte Chelia son factores que parecen atenuar esta fuerte correlación anillo-clima $y$, por consiguiente, los efectos del déficit hídrico responsables del declive del bosque se reducen considerablemente

Palabras clave: Cedrus atlantica, cedro del Atlas, declive, relaciones anillo-clima, Aurès, Argelia.

\footnotetext{
Cedrus atlantica,

l'Atlas, dépérissement, relations cerneclimat, Aurès, Algérie.
} 


\section{Introduction}

La région méditerranéenne représente l'une des 34 zones sensibles de la biodiversité identifiées au niveau mondial. Elle n'abrite pas moins de 10 zones régionales et 52 refuges putatifs (Véla et Benhouhou, 2007).

Les forêts méditerranéennes constituent des écosystèmes de grandes valeurs écologique, génétique et socio-économique. Ces écosystèmes hautement anthropisés, dont le niveau de biodiversité est élevé, font de la Méditerranée une des régions les plus menacées de la planète par les changements globaux (Quézel et Médail, 2003). Ces biomes « en crise " sont considérés comme des modèles clés pour étudier les effets des changements climatiques sur les processus écosystémiques. Les modèles climatiques, développés pour ces régions à l'horizon 2100, prévoient généralement une forte augmentation de la température moyenne annuelle, une diminution de l'ordre de $30 \%$ des précipitations annuelles et enfin une récurrence des événements extrêmes (Jacq, 2008).

Selon Quézel (1998), certaines cédraies d'Afrique du Nord semblent être mises en péril. Cette étude caractérise ces cédraies comme étant dans un état de survie et estime qu'elles finiront par disparaître dans les prochaines décennies. La modélisation adoptée par Demarteau et al. (2007) fait ressortir un scénario selon lequel l'aire de distribution du cèdre de l'Atlas serait considérablement modifiée dans les prochaines années : le climat de l'Afrique du Nord deviendrait trop chaud pour le bon développement du cèdre, son aire de distribution s'en verrait ainsi réduite, et contraindrait donc cette espèce à se confiner dans des zones refuges situées à haute altitude. Par le passé, suite à la période de sécheresse prolongée qui s'est produite de 1872 à 1882, Lapie (1909) a mis en avant une mortalité massive des cédraies méridionales de l'Algérie, telles que celles du Belezma, du Chélia et de Theniet El Had. Suite à un second épisode de sécheresse ayant débuté à la fin des années 1970, qui s'est ensuite accentué durant les dernières décennies (Abdessemed, 1981 ; Messaoudène et al., 2013), les études récentes montrent que ces formations forestières exposées aux bioclimats subhumide et semi-aride font état d'un dépérissement intense. Les diverses conclusions retenues, qu'elles soient limitées au cèdre ou s'étendent à l'échelle globale des forêts méditerranéennes, ne mettent pas en évidence l'impact d'un facteur bien individualisé. Il semblerait que plusieurs paramètres comme les facteurs prédisposants (âge des arbres, potentiel génétique, édaphique...), les facteurs déclenchants (sécheresse, attaques d'insectes défoliateurs...) et les facteurs aggravants (insectes xylophages, champignons parasites...) jouent un rôle conjoint dans le dépérissement des zones forestières méditerranéennes (Sbabdji et al., 2009, 2015 ; Aoubouazza, 2017 ; Sarmoum et al., 2019).

D'après les renseignements recueillis au sein de l'administration des forêts et confirmés par nos observations sur le terrain, les cédraies du massif des Aurès (Belezma et Chélia) sont les plus touchées en Algérie ; elles sont soumises à un dépérissement massif depuis la fin des années 1980. Dans les autres cédraies du pays, comme dans les parcs nationaux de Theniet El Had et du Djurdjura, le phénomène est partiel et se limite au versant sud. Si l'on se réfère à la position sociale des arbres dépéris sur pied et à l'état de la régénération naturelle, il apparaît que le phénomène de dépérissement ne s'apparente pas aux mêmes causes à l'échelle de toutes les cédraies d'Algérie. Au Chélia, par exemple, seuls les arbres très âgés sont atteints par ce phénomène ; les autres stades d'évolution de la futaie à cèdre, de gaulis à perchis, sont épargnés. Ce n'est pas le cas au Belezma où le dépérissement est marqué par une mortalité massive.

Comprendre la résilience des forêts et la réponse aux changements climatiques a des implications importantes pour la gestion et la sauvegarde de ces forêts vulnérables. Cette contribution s'inscrit dans le contexte de la variabilité climatique qui s'opère en Algérie au cours de ce dernier siècle. Le but recherché consiste à vérifier, par une approche dendrochronologique, l'hypothèse selon laquelle les épisodes successifs de sécheresse constatés à partir de la seconde moitié du $X X^{\text {e }}$ siècle auraient eu un impact sur la croissance radiale du cèdre de l'Atlas et son dépérissement au sein des massifs du Belzema et du Chélia (région des Aurès). Par cette approche, on tentera aussi d'apprécier dans quelle mesure les facteurs écologiques propres à ces deux massifs forestiers seraient responsables dans l'amplification ou l'atténuation de ce phénomène. Au final, l'idée est de proposer des solutions afin de réduire au mieux la mortalité survenue au sein de ces formations.

\section{Matériel et méthodes}

\section{Milieu d'étude}

Les cédraies étudiées sont des formations végétales naturelles et se localisent sur deux sites de la région des Aurès : les massifs du Belezma et du Chélia (figure 1). En dépit de leur proximité géographique, ces deux milieux d'étude distants d'environ $60 \mathrm{~km}$ sont très contrastés du point de vue de l'intensité de dépérissement, du degré d'anthropisation, de l'équilibre écologique, du taux de régénération, de l'état sylvicole et du type de sol (Alileche, 2012).

La cédraie du Belezma est située dans le Parc national du Belezma. Elle s'étend sur une superficie de 7000 ha et se présente comme une formation sylvicole très anthropisée et en mauvais état de conservation. Un dépérissement intense y est enregistré, dépassant les $90 \%$ dans certaines stations (photo 1) (Kherchouche et al., 2013). Ces peuplements équiennes présentent une régénération naturelle très faible voire inexistante dans certaines parties du massif. Les sols de ces cédraies sont maigres et peu évolués, de types bruns calcaires et rendzines dolomitiques (Harfouche et Nedjahi, 2003). Le massif est caractérisé par une pluviosité moyenne de $346 \mathrm{~mm} / a n$ dont une partie se précipite sous forme neigeuse (45 jours/an). L'humidité relative est estimée à $60,5 \%$. La température moyenne annuelle ne dépasse pas $15,1^{\circ} \mathrm{C}$ (Alileche, 2012). 
Bois et Forêts des Tropiques - ISSN: L-0006-579X

46 Volume $350-4^{\text {th }}$ quarter - December 2021 - p. 43-56

46 FOCUS / CLIMATE AND RADIAL GROWTH IN CEDRUS ATLANTICA

D’une superficie estimée à 8000 ha, la cédraie du Chélia est du type jardiné (photo 2). C'est une formation bienvenante avec de meilleures aptitudes à occuper des territoires potentiellement appropriés. Le dépérissement s'y limite à quelques sujets âgés. La régénération est remarquable, notamment dans les trouées d'abattage et les remblais de talus. Les sols sont du type rendzine, profonds et évolués avec une litière très abondante. Les signes d'anthropisation sont très discrets (Bentouati, 2008). La moyenne des précipitations annuelles est de l'ordre de 533,6 mm. La neige y est plus abondante (57 jours). On enregistre une température moyenne mensuelle de $16,6{ }^{\circ} \mathrm{C}$. L'humidité relative moyenne enregistrée est de $56 \%$ avec, cependant, des fluctuations considérables durant l'année (40\% à $80 \%$ ) (Alileche, 2012).

Dans chaque site, un choix de trois stations d'échantillonnage est établi sur la base de la distinction entre leurs

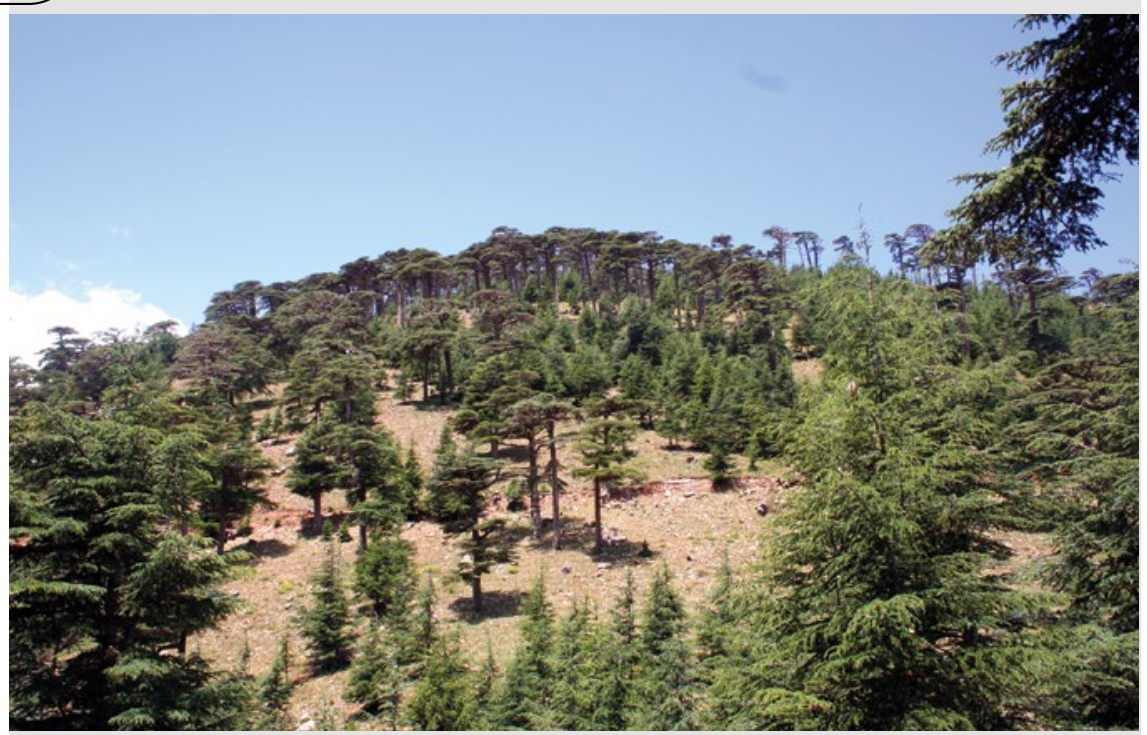

Photo 2.

Aperçu de la cédraie du Chélia avec sa structure jardinée.

Overview of the cedar grove of Chélia with its garden structure.

Photo M. Tafer.

caractéristiques écologiques comme l'altitude, l'exposition, la pente, le type de sol, la densité du peuplement et son degré de dépérissement représenté par le nombre de sujets dépéris sur le nombre total d'arbres de la station (tableau I).

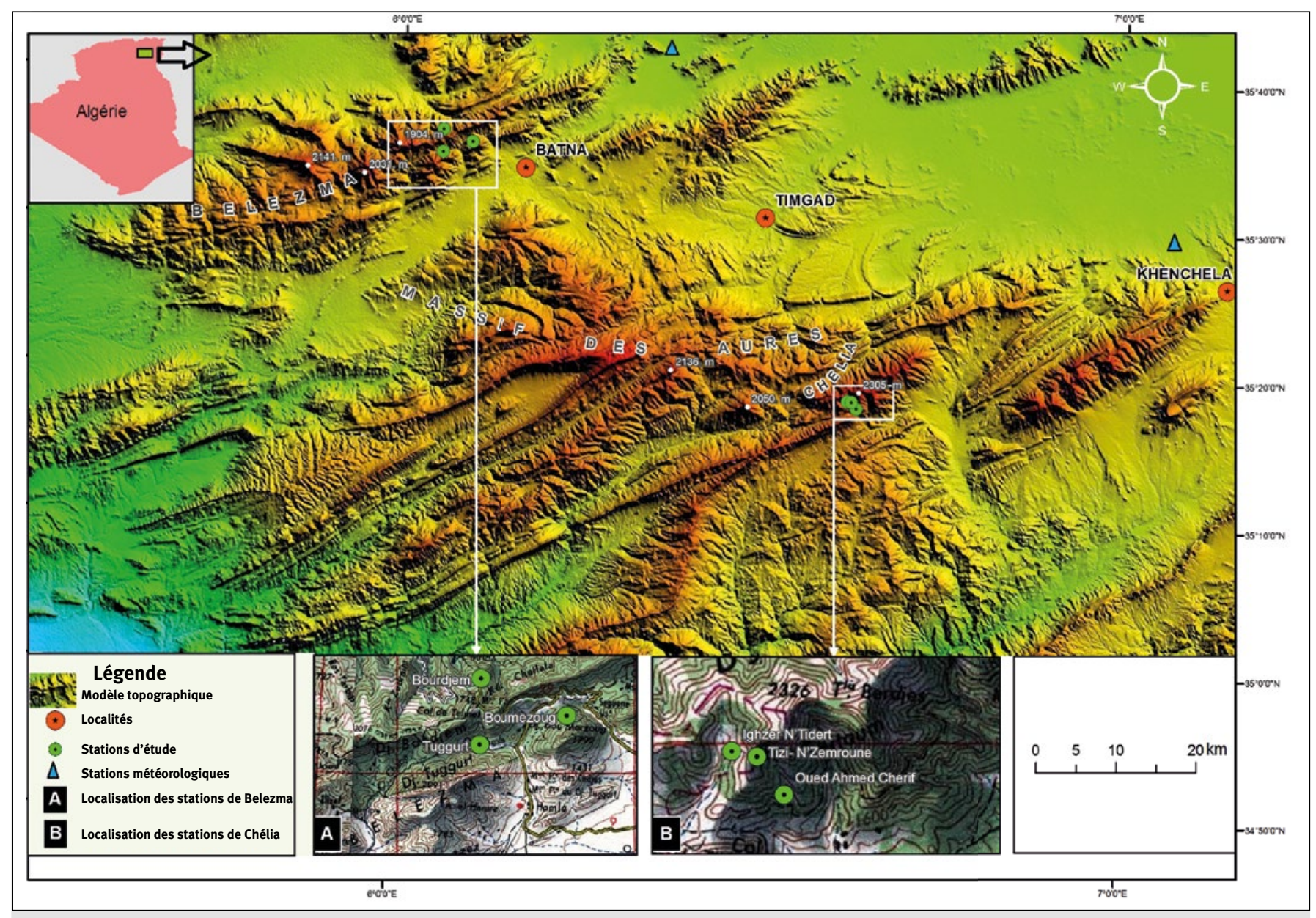

Figure 1.

Carte de situation des deux sites d'étude.

Location map of the two study sites. 
Tableau I.

Caractéristiques écologiques des sites d'étude.

Ecological characteristics of the study sites.

\begin{tabular}{|c|c|c|c|c|c|c|c|c|c|c|}
\hline Sites & Stations & $\begin{array}{l}\text { Latitude } \\
(\mathrm{N})\end{array}$ & $\begin{array}{l}\text { Longitude } \\
\text { (E) }\end{array}$ & Exposition & $\begin{array}{l}\text { Altitude } \\
\text { (m) }\end{array}$ & Bioclimat & $\begin{array}{l}\text { Pente } \\
\text { (\%) }\end{array}$ & $\begin{array}{l}\text { Densité } \\
\text { (N/5 ares) }\end{array}$ & $\begin{array}{c}\text { Dépérissement } \\
\text { (\%) }\end{array}$ & $\begin{array}{c}\text { Type de sol } \\
\text { (Abdessemed, 1981) }\end{array}$ \\
\hline \multirow[t]{3}{*}{ Belezma } & Boumerzoug & $35^{\circ} 35^{\prime} 28^{\prime \prime}$ & $6^{\circ} 05^{\prime} 43^{\prime \prime}$ & Nord & 1500 & $\begin{array}{l}\text { Semi-aride } \\
\text { froid }\end{array}$ & $35-40$ & 16 & 70 & $\begin{array}{l}\text { Sols bruns } \\
\text { calcaires }\end{array}$ \\
\hline & Tuggurt & $35^{\circ} 34^{\prime} 72^{\prime \prime}$ & 6०03'29" & Nord-Est & 1300 & $\begin{array}{l}\text { Semi-aride } \\
\text { froid }\end{array}$ & $25-30$ & 17 & 65 & $\begin{array}{l}\text { Rendzines } \\
\text { décalcarisées }\end{array}$ \\
\hline & Bordjem & $35^{\circ} 36^{\prime} 14^{\prime \prime}$ & $6^{\circ} 03^{\prime} 16^{\prime \prime}$ & Crête & 1750 & $\begin{array}{l}\text { Subhumide } \\
\text { froid }\end{array}$ & $05-10$ & 23 & 35 & $\begin{array}{l}\text { Sols bruns peu } \\
\text { calcaires }\end{array}$ \\
\hline \multirow[t]{3}{*}{ Chélia } & $\begin{array}{l}\text { Oued Ahmed } \\
\text { Chérif }\end{array}$ & $35^{\circ} 18^{\prime} 01^{\prime \prime}$ & 6038’05” & Sud & 1770 & $\begin{array}{l}\text { Semi-aride } \\
\text { froid }\end{array}$ & $40-50$ & 18 & 25 & $\begin{array}{l}\text { Sols bruns } \\
\text { peu calcaires }\end{array}$ \\
\hline & $\begin{array}{l}\text { Ighzer } \\
\text { N'Tidert }\end{array}$ & $35^{\circ} 18^{\prime} 31^{\prime \prime}$ & 6037'16" & Est & 1900 & $\begin{array}{l}\text { Subhumide } \\
\text { froid }\end{array}$ & $30-35$ & 22 & 20 & $\begin{array}{l}\text { Rendzines } \\
\text { décalcarisées }\end{array}$ \\
\hline & $\begin{array}{l}\text { Tizi- } \\
\text { N’Zemroune }\end{array}$ & $35^{\circ} 18^{\prime} 27^{\prime \prime}$ & 6037'37" & Ouest & 1990 & $\begin{array}{l}\text { Subhumide } \\
\text { froid }\end{array}$ & 10 & 20 & 20 & $\begin{array}{l}\text { Rendzines } \\
\text { décalcarisées }\end{array}$ \\
\hline
\end{tabular}

\section{Données climatiques}

Les données météorologiques exploitées sont les cumuls mensuels des précipitations (enregistrés de 1936 à 2009) et les températures moyennes mensuelles (enregistrées de 1960 à 2009). Pour le mont Chélia, celles-ci proviennent du poste météorologique de Khenchela, situé à une altitude de $1150 \mathrm{~m}$ et à environ $40 \mathrm{~km}$ du site d'étude. Concernant le massif du Belezma, elles sont recueillies au niveau de la station météorologique de Batna (1 $045 \mathrm{~m}$ d'altitude), distante de $30 \mathrm{~km}$ de notre aire d'échantillonnage, et complétées par les valeurs de Climate Research Unit (CRU) version 3.24 (Harris et al., 2014).

L'indice standardisé de précipitations (Standardised Precipitation Index, "SPI ») a été utilisé pour quantifier les tendances dans cette série de données. Cet indice a été élaboré en vue de caractériser les déficits de précipitations pour une période donnée. Une application développée par le National Drought Mitigation Center ${ }^{1}$ a permis de générer les valeurs de l'indice SPI des deux sites. Cet indice correspond à la transformation de la série temporelle des précipitations en une distribution normale standardisée de moyenne nulle et d'écart-type unitaire, et prend en considération l'importance du temps dans l'analyse de la disponibilité des ressources en eau (McKee et al., 1993). Kherchouche et al. (2013) considèrent qu'une année est extrêmement sèche lorsque le cumul de ses précipitations d'octobre à juin est inférieur au seuil de $80 \%$ de la moyenne. Quant à Hirche et al. (2007), ils définissent une année sèche et une année humide d'après l'écart à la moyenne. Elle sera sèche si la valeur est en deçà de 10 \%, c'est-à-dire inférieure à $90 \%$ de la moyenne, et humide au-delà. Pour notre part, les années sèches et humides sont déterminées par les valeurs de leur SPI classées selon la nomenclature établie par McKee et al. (1993) et rapportée dans le tableau II. Une courbe moyenne mobile de quatre ans a permis une meilleure expression de la tendance de la courbe des valeurs SPI.

L'appréciation de l'évolution des températures au cours de ces dernières décennies est rendue possible grâce à l'exploitation des températures moyennes annuelles standardisées en soustrayant la moyenne et en divisant par l'écart-type (Cuccia, 2013).

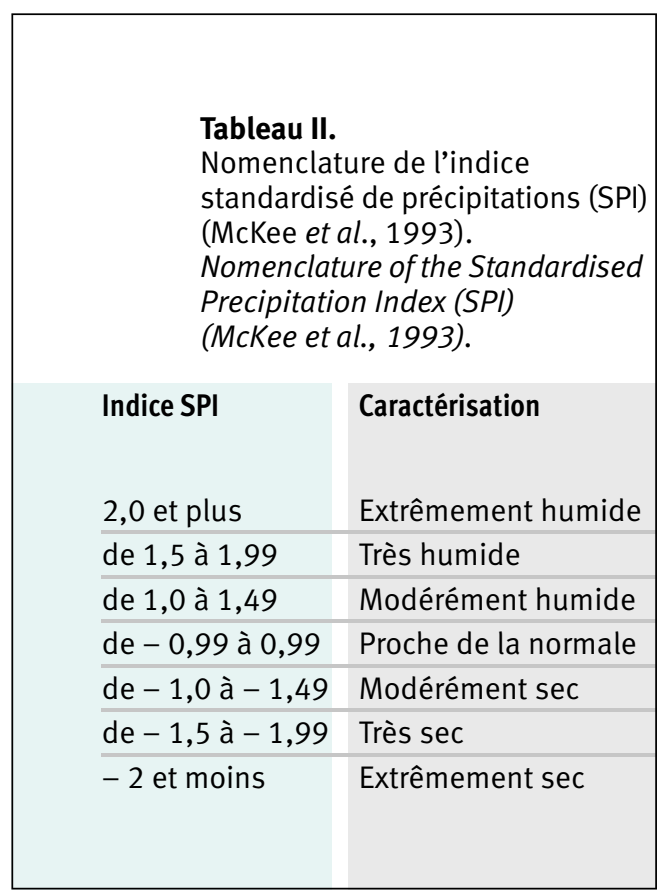




\section{Échantillonnage et analyses statistiques}

En se basant sur l'approche dendrochronologique, dans chaque site étudié, 60 arbres (20 arbres par station) ont été étudiés. La sélection de ces arbres s'est faite de manière à être orientée vers des arbres sains avec des catégories d'âge variées, et en évitant les sujets situés près des cours d'eau ou des sources afin d'augmenter le signal climatique enregistré dans les cernes de croissance et de minimiser les effets d'autres facteurs. Deux carottes (orientations opposées) ont été prélevées sur chaque arbre, à cœur et à hauteur de la poitrine (1,30 m), à l'aide d'une tarière de Pressler (photo 3). Les carottes ont été poncées et interdatées selon les principes de base de la dendrochronologie (Stokes et Smiley, 1996). L'épaisseur des cernes a été mesurée avec une précision de $1 / 1000 \mathrm{~mm}$ à l'aide d'un banc de mesure semi-automatique LINTAB $^{\text {tm5 }}$. Le programme COFECHA (Holmes, 1983) a été utilisé pour le contrôle statistique de la qualité de l'interdatation.

Afin de réduire au maximum les effets et les tendances non liés à la variation climatique, une standardisation des séries chronologiques interdatées a été effectuée avec le programme ARSTAN sous Windows (Cook, 1985), en utilisant une fonction spline cubique de lissage avec une fenêtre définie à $67 \%$ de la longueur de la série (Cook, 1985 ; Cook et al., 1995 ; Delwaide et Filion, 2010). Enfin, les séries détendancées qui en résultent ont été soumises à un traitement ultime permettant de retirer l'autocorrélation résiduelle et d'éliminer ainsi les phénomènes d'arrièreeffets. En définitive, une chronologie résiduelle avec une variabilité relativement homogène dans le temps et une moyenne égale à 1 en résulte (Fritts, 1976).

Les principaux paramètres statistiques utilisés pour la caractérisation du signal climatique enregistré dans les séries de cernes sont : l'écart-type (ET), la sensibilité

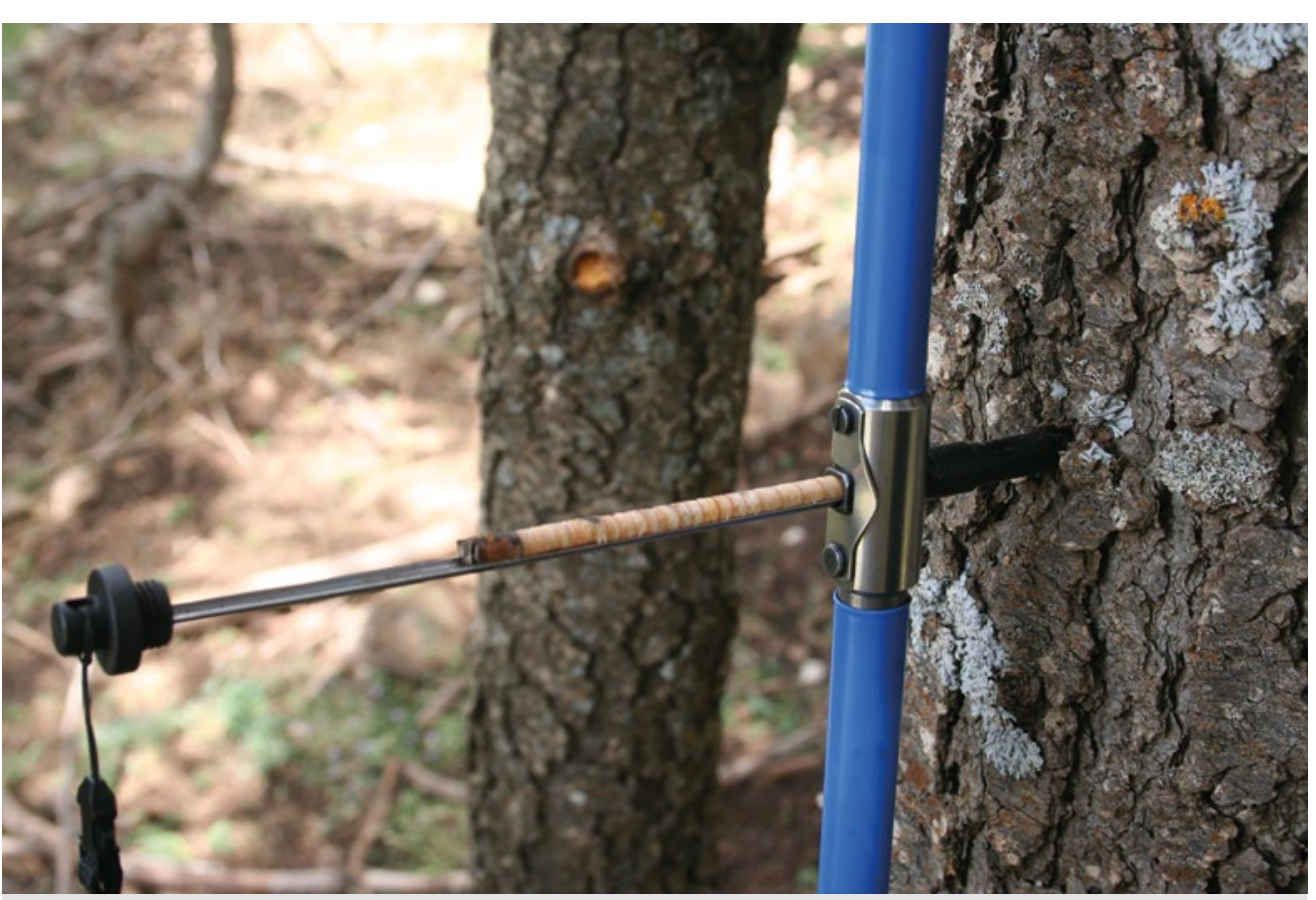

Photo 3.

Prélèvement sur chaque arbre, à cœur et à hauteur de la poitrine $(1,30 \mathrm{~m})$ à l'aide d'une tarière de Pressler.

Sampling of each tree at heart and chest height $(1.30 \mathrm{~m})$ using a Pressler auger. Photo M. Tafer. moyenne (SM), l'autocorrélation d'ordre 1 (AC1), la variance du premier vecteur propre (VP1), la corrélation moyenne entre carottes (CMC), et le signal exprimé dans la population (EPS : Expressed Population Signal). La sensibilité moyenne (SM) permet de définir la variation moyenne entre deux épaisseurs annuelles successives. Elle exprime l'ampleur des changements affectant, à court terme, l'épaisseur du cerne (Lebourgeois et Mérian, 2012).

L'autocorrélation d'ordre 1 estime l'interdépendance de deux cernes successifs de la même série chronologique. Elle quantifie l'effet de la persistance liée aux conditions conduisant à l'élaboration du cerne de l'année $t-1$ sur la mise en place du cerne de l'année $t$ (Lebourgeois et Mérian, 2012). La variance du premier vecteur propre reflète la qualité du signal climatique contenu dans les cernes de croissance (Slimani et al., 2014).

L'EPS constitue un très bon paramètre pour apprécier la robustesse des chronologies utilisées et quantifie la force relative du signal, commun à tous les arbres par rapport au signal total. Ses valeurs proches de 1 traduisent un bruit réduit et un signal fort de la population échantillonnée (Lebourgeois et Mérian, 2012).

La méthode Neuwirth (Neuwirth et al., 2007) a été adoptée pour déceler les années caractéristiques (AC) correspondant aux réactions de croissance annuelles dues aux changements brusques des conditions environnementales (Schweingruber et al., 1990). Cette méthode a consisté à transformer les séries chronologiques moyennes brutes des populations étudiées en séries d'indice Cropper (ICr). Ce dernier correspond au rapport de la largeur du cerne d'une année à la moyenne des largeurs mesurées sur une fenêtre de 13 années centrée sur l'année considérée. Les chronologies $\mathrm{ICr}$ qui en résultent sont tronquées de six années au niveau des deux extrémités, puis normalisées pour avoir une moyenne de zéro et un écarttype de un. Les valeurs obtenues sont rangées en trois classes de déviation de croissance positive (cerne épais) ou négative (cerne mince) : faible $(\mathrm{ICr}>1)$, forte $(\mathrm{ICr}>1,28)$ et extrême $(\mathrm{ICr}>1,645)$. Les $\mathrm{ICr}$ des cernes épais correspondent aux AC positives ou humides. Inversement, on associe les $\mathrm{ICr}$ des cernes minces aux AC négatives ou sèches.

La relation cerne-climat a été évaluée au moyen de corrélations saisonnières établies par le programme Seascorr (Meko et al., 2011). Les fichiers input sont la série chronologique des indices de croissance et les 24 régresseurs climatiques des précipitations mensuelles et températures moyennes mensuelles d'octobre de l'année $t-1$ à septembre de l'année $t$. 


\section{Résultats}

\section{Analyse des données climatiques}

En se référant au tableau III des valeurs de l'indice SPI calculées sur la période commune (1961-2009), nous constatons que le Belezma a enregistré pas moins de 14 années de xéricité prononcée, réparties à parts égales entre sept années « modérément sèches » et sept autres années " très sèches ». En revanche, au Chélia, on dénombre seulement huit années dont sept « sèches » et une seule qualifiée de " très sèche ». Inversement, les valeurs SPI correspondant aux périodes humides penchent nettement en faveur du Chélia. Sur un total de huit années pluvieuses, six s'inscrivent dans la catégorie "modérément humides » et deux dans celle "très humides ". Ces années pluvieuses se font par contre plus discrètes dans le Belezma : quatre années réparties équitablement entre " modérément humides » et " très humides ».

La courbe de lissage de moyenne mobile de l'indice SPI du Belezma (1910-2009) (figure 2) montre une nette tendance à la baisse dès le début de la décennie 1960. En effet, les indices SPI enregistrés au cours de ces six dernières décennies sont dans leur majorité (75\%) de signe négatif. Par ailleurs, durant cette même période, ce massif dévoile aussi deux épisodes prolongés de sécheresse carac-

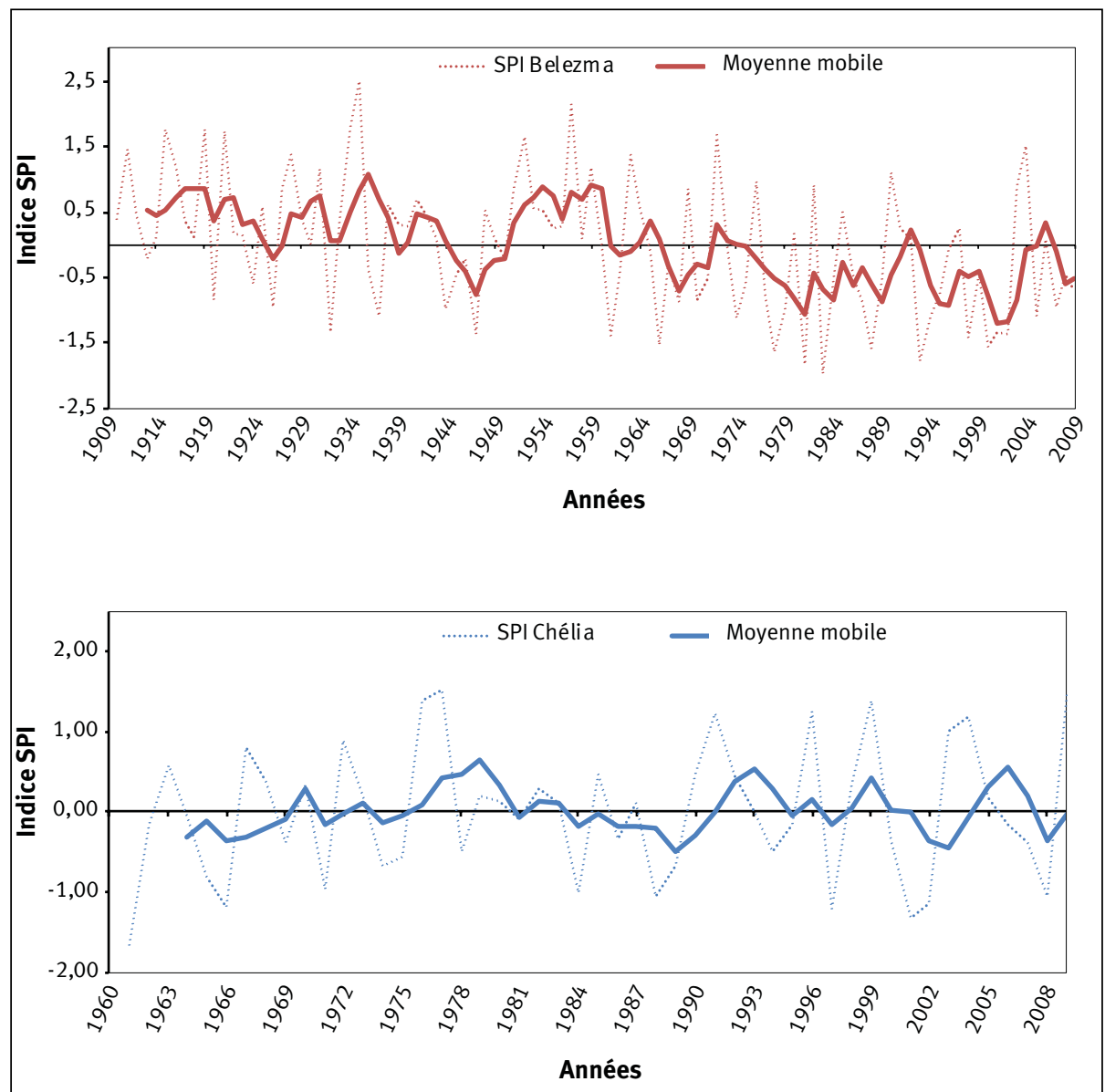

Figure 2 .

Valeurs de l'indice standardisé de précipitations (SPI) pour les massifs du Belezma (1910-2009) et du Chélia (1960-2009).

Standardized precipitation index (SPI) values for the Belezma (1910-2009) and Chélia (1960-2009) massifs. térisés par des années à indices SPI négatifs : le premier épisode s'étale sur environ 16 années (1974-1989) et affiche un effectif de quatre années « très sèches » $(1978,1981$, 1983 et 1988) et le second a été enregistré peu de temps après, entre 1993 et 2002, avec huit années affichant un SPI négatif dont cinq années qualifiées de «très sèches ». Ce dernier épisode a été plus sévère que le premier en raison de l'apparition de successions climatiques de deux années « très sèches ", 1993-1994, avec des valeurs SPI respectives de $-1,77$ et $-1,70$, et de trois autres années considérées comme " modérément sèches ", 2000-2001-2002, ayant respectivement des indices SPI de $-1,56,-1,35$ et $-1,37$. Cependant, si on se base sur un indice SPI calculé sur la période couvrant les mois de mars à juin, qui présentent une relation significative entre la précipitation et l'activité cambiale dans la région des Aurès, on constate que le déficit hydrique se restreint uniquement aux années 2001 et 2002, identifiées respectivement comme « modérément sèche » et « très sèche ». En revanche, l'année 2000 se décline comme proche de la normale.

Ces deux épisodes de décrochage sont également observés dans le Chélia mais avec une plus courte durée et une intensité moindre. Le premier se situe entre 1984 et 1991 : les années $1984(\mathrm{SPI}=-1)$ et $1988(\mathrm{SPI}=-1,04)$ y sont identifiées comme "modérément sèches ». Le second épisode est considérablement plus court (2001-2004) mais légèrement plus prononcé : les années $2001(\mathrm{SPI}=-1,32)$ et 2002 (SPI = - 1,14) y sont identifiées comme " modérément sèches ». Cependant, en se référant à l'indice SPI limité à la saison de croissance, l'année 2002 s'avère plutôt « très sèche ».

Tableau III.

Nombre d'événements climatiques extrêmes dans les deux sites d'étude (1961-2009). Number of extreme climatic events at the two study sites (1961-2009).

\begin{tabular}{|c|c|c|}
\hline SPI & Belezma & Chélia \\
\hline$\geq 2,0$ & 0 & 0 \\
\hline 1,5 à 1,99 & 2 & 2 \\
\hline 1,0 à 1,49 & 2 & 6 \\
\hline - 0,99 à 0,99 & 32 & 33 \\
\hline$-1,0$ à - 1,49 & 7 & 7 \\
\hline$-1,5$ à $-1,99$ & 7 & 1 \\
\hline$\leq-2$ & 0 & 0 \\
\hline $\begin{array}{l}\text { SPI : inc } \\
\text { précipit }\end{array}$ & $\begin{array}{l}\text { ce standarc } \\
\text { tions. }\end{array}$ & de \\
\hline
\end{tabular}


Bois et Forêts des Tropiques - ISSN: L-0006-579X

Volume $350-4^{\text {th }}$ quarter - December 2021 - p. 43-56

La figure 3 montre que les températures moyennes annuelles standardisées ont enregistré un accroissement significatif $(\mathrm{p}<0,001 ; \mathrm{n}=50)$ à partir des années 1970. la plus faible étant enregistrée à Ighzer N’Tidert $(0,255)$. La sensibilité moyenne enregistrée fluctue entre 0,339 à Ighzer N'Tidert et 0,469 à Oued Ahmed Chérif.

La variance du premier vecteur propre (VP1) et la corrélation moyenne entre carottes (CMC) de chaque population sont élevées dans toutes les stations. La plus faible valeur de VP1 (65,73\%) est transcrite à Ighzer N'Tidert et la plus élevée $(79,26 \%)$ à Oued Ahmed Chérif. La CMC oscille entre les valeurs de 0,637 et 0,783 , enregistrées respectivement à Ighzer N’Tidert et Oued Ahmed Chérif.

Les stations de Tuggurt (massif du Belezma) et de Oued Ahmed Chérif (massif du Chélia) ont enregistré à la fois les ET, SM, CMC et VP1 les plus élevés. Pour autant, les quatre paramètres ont enregistré leurs plus faibles valeurs, dans les mêmes sites, à Boumerzoug (Belezma) et Ighzer N'Tidert (Chélia).

La chronologie moyenne du Chélia affiche une SM plus élevée que celle du

\section{Analyse de la croissance radiale}

L'analyse de la croissance radiale et l'appréhension de la relation cerne-climat reposent sur les chronologies résiduelles, qui affichent les meilleures valeurs des paramètres statistiques pour une analyse dendroclimatique (tableau IV). La longueur des chronologies finales est basée sur le seuil EPS de 0,85 .

On remarque que les valeurs de l'écart-type (ET) et de la sensibilité moyenne (SM) sont variables entre les stations et les deux sites d'étude. La plus grande dispersion de l'indice de croissance autour de la moyenne, exprimée par la valeur de l'écart-type, caractérise la population de Tuggurt $(0,387)$,
Belezma. Néanmoins, une analyse sur la période commune (1936-2009) révèle que ce paramètre est plus fort au Belezma $(0,468)$. Les valeurs VP1 et CMC sont plutôt comparables entre les deux sites.

La figure 4 montre un bon synchronisme des deux chronologies globales. Les valeurs minimales de l'indice de croissance, enregistrées simultanément en 2001, sont de 0,240 au Belezma et de 0,197 au Chélia. Les maximums obtenus sont de 2,145 (1996) au Belezma et de 1,457 (1943) au Chélia. De fortes fluctuations de cet indice sont perceptibles à partir des années 1950 et s'accentuent à la fin du siècle avec de nombreux cernes minces. Sur la période commune, le Belezma a enregistré une plus forte variabilité de la croissance radiale.

Tableau IV.

Paramètres statistiques des chronologies résiduelles.

Statistical parameters of residual chronologies.

\begin{tabular}{|c|c|c|c|c|c|c|c|c|c|c|c|c|}
\hline \multirow[t]{5}{*}{ Sites } & \multirow{3}{*}{$\begin{array}{l}\text { Stations } \\
\text { Boumerzoug }\end{array}$} & \multirow{3}{*}{$\begin{array}{c}\text { Durée } \\
\text { 1879-2009 }\end{array}$} & \multirow{3}{*}{$\begin{array}{c}\text { I } \\
0,982\end{array}$} & \multirow{3}{*}{$\begin{array}{c}\text { ET } \\
0,327\end{array}$} & \multirow{3}{*}{$\begin{array}{c}\text { SM } \\
0,352\end{array}$} & \multirow{3}{*}{$\begin{array}{c}\text { SK } \\
0,672\end{array}$} & \multirow{3}{*}{$\begin{array}{c}\text { KU } \\
1,334\end{array}$} & \multirow{3}{*}{$\begin{array}{l}\text { AC1 } \\
0,019\end{array}$} & \multirow{3}{*}{$\begin{array}{l}\text { VP1 } \\
\text { (\%) } \\
70,51\end{array}$} & \multirow{3}{*}{$\begin{array}{c}\text { CMC } \\
0,686\end{array}$} & \multicolumn{2}{|c|}{ EPS > 0,85} \\
\hline & & & & & & & & & & & iode & $\begin{array}{l}\text { Nombre } \\
\text { d'arbres }\end{array}$ \\
\hline & & & & & & & & & & & 1903-2009 & 3 \\
\hline & Tuggurt & $1867-2007$ & 0,993 & 0,387 & 0,461 & 0,383 & 2,077 & $-0,118$ & 79,23 & 0,779 & $1870-2007$ & 2 \\
\hline & Bordjem & $1912-2009$ & 0,994 & 0,318 & 0,396 & $-0,125$ & 1,262 & $-0,115$ & 70,73 & 0,691 & $1915-2009$ & 3 \\
\hline \multirow[t]{5}{*}{ Belezma } & & $1867-2009$ & 0,989 & 0,289 & 0,338 & 0,143 & 2,035 & $-0,132$ & 67,88 & 0,669 & $1876-2009$ & 3 \\
\hline & & $1936-2009$ & 0,989 & 0,356 & 0,468 & $-0,010$ & 0,814 & $-0,146$ & 67,68 & 0,667 & $1936-2009$ & 3 \\
\hline & Oued Ahmed Chérif & $1936-2009$ & 1,000 & 0,341 & 0,469 & $-0,644$ & 0,379 & $-0,107$ & 79,26 & 0,783 & $1942-2009$ & 2 \\
\hline & Ighzer N'Tidert & $1958-2009$ & 0,995 & 0,255 & 0,339 & $-0,995$ & 1,389 & $-0,174$ & 65,73 & 0,637 & 1936-2009 & 3 \\
\hline & Tizi-N’Zemroune & $1955-2009$ & 1,006 & 0,324 & 0,434 & $-1,151$ & 1,178 & $-0,084$ & 76,61 & 0,756 & $1959-2009$ & 2 \\
\hline hélia & & $1936-2009$ & 0,998 & 0,298 & 0,407 & $-0,750$ & 0,206 & $-0,140$ & 68,60 & 0,667 & 1944-2009 & 3 \\
\hline
\end{tabular}

$\mathrm{I}$ : indice de croissance moyen ; ET : écart-type ; SM : sensibilité moyenne ; SK : coefficient d'asymétrie (skewness) ; $\mathrm{KU}$ : coefficient d'aplatissement (kurtosis) ; AC1 : autocorrélation d'ordre 1 ; VP1 : variance du $1^{\text {er }}$ vecteur propre ;

CMC : corrélation moyenne entre carottes ; EPS : signal exprimé dans la population (expressed population signal). 


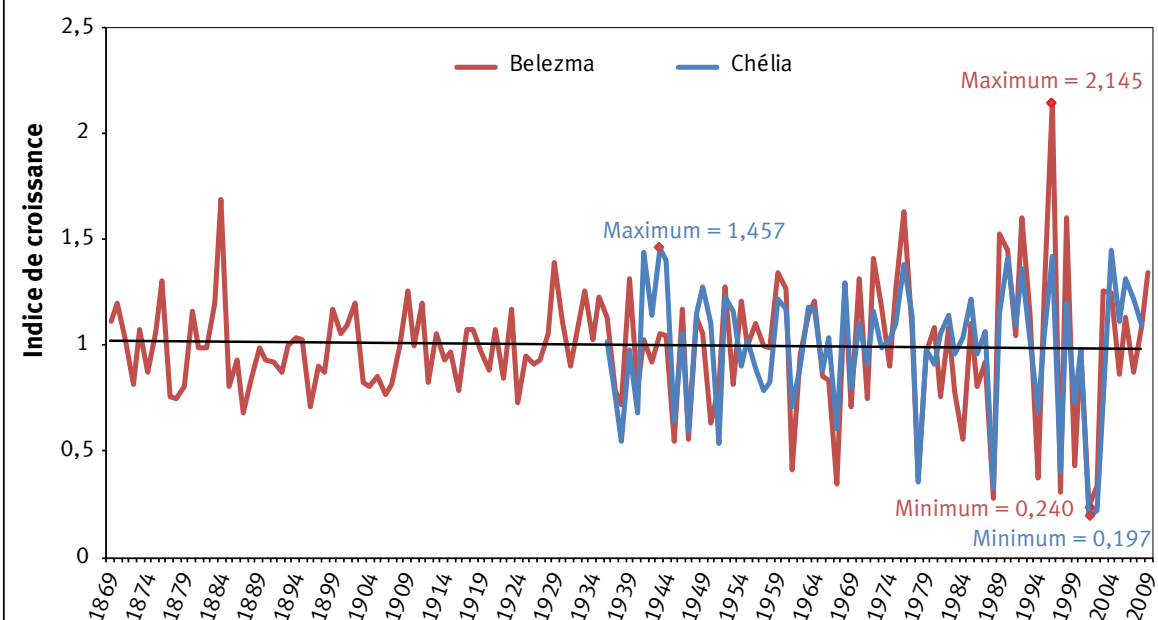

Années

Figure 4.

Variations interannuelles de l'indice de croissance des deux sites d'étude.

Interannual variations in the growth index of the two study sites.

\section{Réponse aux extrêmes climatiques}

La comparaison des indices Cropper ( $\mathrm{ICr}$ ) des chronologies maîtresses, sur la période commune 1964-2009, correspondant à la plus jeune station (Ighzer N’Tider), révèle une différence du nombre des $A C$ enregistré au sein des deux cédraies étudiées. Les stations du Belezma comptabilisent un effectif des AC variant de 16 à 18 ; celles du Chélia oscillent entre 10 et 12 années (figure 5). Tuggurt (massif du Belezma) est incontestablement la station la plus marquée par les extrêmes climatiques secs. On y dénombre $11 \mathrm{AC}$ négatives dont six sont considérées comme "extrêmes ». En revanche, Ighzer N’Tidert (massif du Chélia), avec cinq AC négatives, est la station la moins touchée par cette sécheresse.

Les $\mathrm{ICr}$ des chronologies globales des deux sites sur la période commune (1964-2009) confirment les différences constatées au niveau des chronologies maîtresses. Le Belezma affiche un nombre de 17 événements caractéristiques alors que le Chélia n'en comptabilise que 12. Néanmoins, les deux sites expriment un synchronisme élevé par rapport à ces évé-
Par ailleurs, l'analyse de la chronologie du Belezma sur la période commune à ses trois stations (1900-2000) fait apparaître une différence de répartition des $A C$ perceptible sur les deux moitiés de ce siècle. En effet, la seconde moitié du XXe siècle comptabilise 18 événements extrêmes, un nombre trois fois supérieur à celui enregistré sur la période 1900-1950, qui n’a connu que six événements extrêmes.

\section{Analyse de la relation cerne-climat}

Les résultats des corrélations saisonnières, calculées sous le programme Seascorr, sont illustrés dans la figure 6 . À l'échelle mensuelle, le Belezma affiche une corrélation significative $(p<0,01)$ et positive de la croissance radiale avec la pluviosité des mois de septembre et octobre de l'année $t-1$ et janvier, février, mars, avril et mai de l'année $t$.

Concernant les températures, seule celle du mois de juin exprime une corrélation négative et significative.

Dans le Chélia, l’impact des précipitations n'est significatif que pour les mois de septembre de l'année $t-1$ et mars de l'année $t$. Vis-à-vis des températures, aucune corrélation significative n'y est perceptible.

Par ailleurs, il y a lieu de signaler que les analyses considérant des précipitations cumulées sur des intervalles de temps supérieurs à un mois ont généré des corrélations de plus en plus similaires entre les deux sites, notamment à partir d'un jeu de données de six mois de précipitations, avec, toutefois, des corrélations légèrement supérieures pour le Belezma.
Figure 5.

Années caractéristiques (AC) basées sur les indices Cropper pour les deux sites d'étude. Les AC faibles sont représentées par des symboles en blanc, les AC fortes en gris, les AC extrêmes en noir, le carré correspond à une $A C$ positive et le cercle à une $A C$ négative. Characteristic years (CA) based on Cropper indices for the two study sites. Weak CAs are represented by symbols in white, strong CAs in grey, extreme CAs in black, the square corresponds to a positive $C A$ and the circle to a negative $C A$.. 


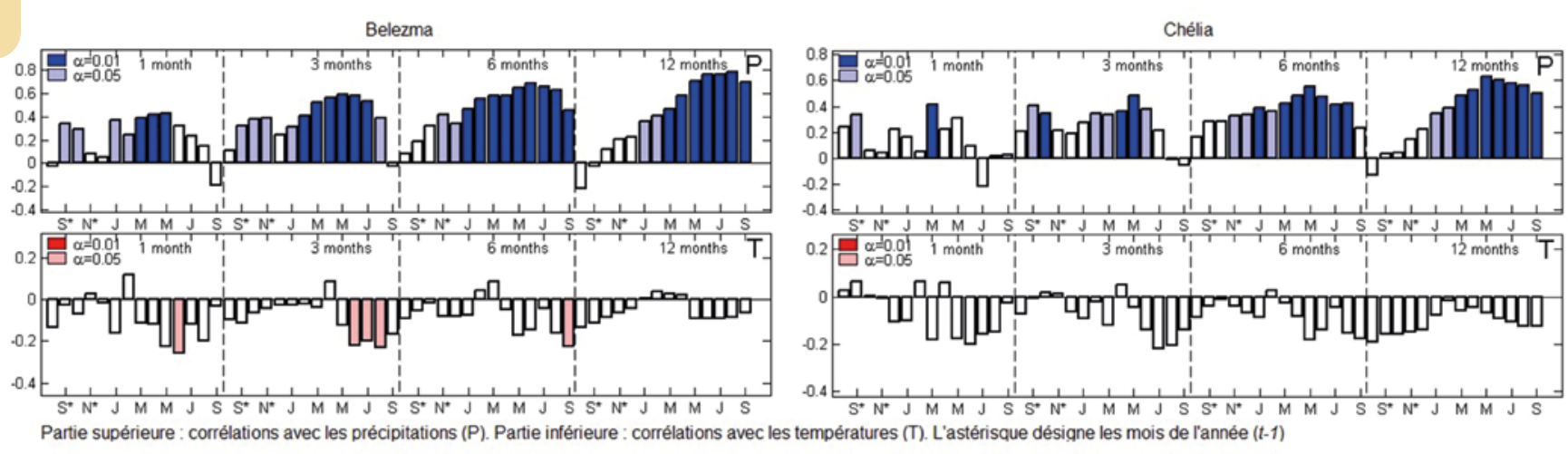

Figure 6.

Corrélations saisonnières entre les indices de croissance des chronologies globales des deux sites d'étude et les variables climatiques saisonnières. En haut les corrélations avec la variable climatique primaire, les précipitations $(P)$. En bas les corrélations avec la variable climatique secondaire, la température (T). L'astérisque $\left.{ }^{\star}\right)$ indique les mois de l'année $t-1$.

Seasonal correlations between the growth indices of the global chronologies of the two study sites and the seasonal climate variables. Top: correlations with the primary climate variable, rainfall $(P)$. Bottom: correlations with the secondary climate variable, temperature (T). The asterisk $\left(^{*}\right)$ indicates the months of year $t-1$.

\section{Discussion}

De l'expression des valeurs SPI calculées (figure 2), il est incontestablement établi que le Belezma constitue le massif forestier le plus affecté par les années de sécheresse. De 1960 à 2009, 14 événements extrêmes de « modérément secs » à « très secs » sont comptabilisés, soit un chiffre supérieur à celui dénombré au Chélia (tableau III). L'origine orographique de la pluviométrie qui caractérise cette région est un élément explicatif à cette différenciation réelle. Les pluies en Algérie sont, en effet, conditionnées par la direction des axes montagneux par rapport à la mer et aux vents humides. Le relief du Chélia, avec ses sommets dépassant $2000 \mathrm{~m}$ d'altitude, constitue un écueil efficace pour les masses d'air chargées d'humidité (Meharzi, 1994).

Sur la courbe lissée des indices SPI de la série climatique centenaire du Belezma (figure 2), une tendance à la baisse dès la décennie 1960 est très visible. Durant cette seconde moitié du XXe siècle, pas moins de $75 \%$ des valeurs SPI sont négatives. Cette péjoration de la pluviosité a été également constatée par Hirche et al. (2007) pour quelques zones arides sahariennes, présahariennes et steppiques des hautes plaines algériennes. Les épisodes de décrochage de la courbe lissée traduisent une forte intensité et fréquence des événements de déficit hydrique au sein des formations sylvatiques du massif du Belezma. Ces épisodes correspondent à deux périodes distinctes : le premier a sévi de 1974 à 1989 (75 \% des SPI < 0), où les années 1978, 1981,1983 et 1988 ont été très sèches. D'ailleurs, c'est au cours de cette période que l'épisode de dépérissement survenu à la fin des années 1970 a été signalé dans l'Aurès (Bentouati, 2008). D'une durée plus courte mais d'un déficit plus intense, le second épisode a été enregistré entre 1993 et 2002. Une sévérité qui est d'autant plus accrue par la succession d'années xériques telles que 1993-1994 et 20012002. Au Chélia, on distingue les mêmes phases de xéricité mais leur persistance et leur intensité sont bien moindres que celles du Belezma.
Selon Allen et al. (2010) et Navarro-Cerrillo et al. (2019), les dernières vagues de dépérissement sont principalement dues aux stress hydrique et thermique enregistrés depuis les années 1970. Cette conjugaison d'événements de déficit hydrique et leur occurrence rapprochée a été fatale aux formations sylvatiques fragiles et vulnérables de ces massifs. Ce dernier épisode de dépérissement a touché de plein fouet les peuplements de cèdre du massif du Belezma, alors qu'il n'a eu qu'un impact superficiel au Chélia (Bentouati, 2008 ; Messaoudène et al., 2013). Les mêmes événements xériques sont rapportés par Touchan et al. $(2008,2017)$ pour tout le Nord-Ouest africain. Dans ce contexte, ces auteurs font remarquer que cette période de sécheresse extrême qui s'est produite dans le Nord-Ouest africain entre la fin du $X X^{e}$ et le début du XXI ${ }^{e}$ siècle est un événement climatique exceptionnel au cours des cinq derniers siècles. Ces mêmes périodes critiques ont été identifiées par Nouaceur et Laignel (2015) pour le littoral et les hautes plaines d'Algérie.

En effet, un tel phénomène est rarement décrit dans la littérature consultée. D’après M'hirit et Benzyane (2006), les sécheresses se produisent en moyenne tous les 11 ans, leur durée est plutôt courte, généralement d'environ un an et demi. Celles qui prévalent sur une durée plus longue sont relativement rares. Ces auteurs rapportent que la sécheresse qui a sévi de 1979 à 1984 au Maroc ne survient qu'une fois tous les quatre siècles et demi.

Les plus faibles valeurs des paramètres analysés, ET, $\mathrm{SM}, \mathrm{CMC}$ et VP1, ont été enregistrées à la station de Ighzer N'Tidert, tandis que les plus élevées sont observées à la station de Oued Ahmed Chérif (tableau IV). La station de Ighzer N'Tidert semble être favorisée par son altitude élevée, son exposition (est) relativement fraîche et ombragée. Au contraire, celle de Oued Ahmed Chérif, située à une basse altitude, se présente sur une forte pente et une exposition sud, ce qui amoindrit les précipitations du nord-ouest et la soumet à un ensoleillement excessif. Les fluctuations des valeurs de ET et SM enregistrées entre les deux cédraies étudiées sont expliquées par la relative hétérogénéité des 
conditions écologiques entre les stations et les deux sites d'étude. Le signal climatique intense enregistré au niveau des stations étudiées et exprimé par des CMC $(\geq 0,637)$ et VP1 ( $\geq 65,73 \%$ ) élevées reflète le bon synchronisme des patrons de croissance entre les stations de chaque site d'étude, suggérant ainsi que les différences mises en exergue par les valeurs des ET sont faibles. On remarque que les sites les plus sensibles (Tuggurt et Oued Ahmed Chérif) expriment la variabilité de la croissance la plus élevée ainsi qu'une plus forte synchronisation des patrons de croissance (CMC), leur permettant ainsi d'enregistrer un meilleur signal climatique (VP1).

En dépit de la forte variabilité climatique installée dès la fin des années 1950, la longueur des chronologies du Belezma a pondéré substantiellement les valeurs de ces paramètres, notamment celui du signal climatique enregistré par les séries temporelles. Dans ce sens, Slimani et al. (2014) ont signalé l'effet de la longueur de la chronologie sur la force du signal climatique enregistré dans les cernes de croissance pour la cédraie de Guetiane, située à l'extrême ouest de la région des Aurès. Les auteurs mettent en évidence la tendance à l'augmentation du synchronisme entre les patrons de croissance des arbres induite par des conditions de plus en plus sèches à partir de la seconde moitié du $X X^{e}$ siècle. En effet, dans la reconstruction de la précipitation d'octobre à juin à partir de la cédraie du Belezma, Kherchouche et al. (2013) rapportent un coefficient de détermination ajusté de l'ordre de 0,66 sur la période 1971-2009, contre un coefficient de 0,57 sur l'intervalle 1931-1970. La pondération de la force du signal climatique sur la période totale de calibration (1931-2009) a été matérialisée par un coefficient de détermination ajusté de l'ordre de 0,59.

Effectivement, en éliminant cet effet de la longueur de la série, le Belezma paraît de loin plus sensible à la variabilité climatique et affiche des valeurs de ET et SM plus élevées. Ceci est confirmé sur la figure 4, qui montre un indice de croissance du Belezma plus dispersé que celui du Chélia. En effet, l'analyse des chronologies globales des deux sites sur une période commune (1936-2009) a révélé une plus forte variabilité de l'indice de croissance du Belezma générée par un signal climatique plus intense et moins stable. Une variabilité climatique qui est caractérisée par des épisodes de déficit hydrique plus sévères et plus fréquents dans ce site. Les résultats révèlent, en effet, que les arbres sondés dans le massif du Belezma (SM $=0,468)$ étaient plus sensibles à la variabilité climatique que ceux du massif du Chélia (SM $=0,407)$. Ces valeurs sont proches de celles rapportées par Kherchouche et al. (2013), Slimani (2014) et Sarmoum et al. (2019) pour les cédraies des Aurès et de Theniet El Had. Les coefficients d'autocorrélation d'ordre 1 presque nuls (de $-0,146$ à - 0,140) dénotent l'élimination de la rémanence des conditions antérieures de croissance persistante dans les chronologies Standard et Arstan. Ceci constitue l'un des critères principaux pour la sélection de la série standardisée à utiliser dans les analyses dendrochronologiques. Lebourgeois et Mérian (2012) considèrent ces autocorrélations basses comme propres aux biotopes soumis à un stress hydrique intense où l'effet persistance d'élaboration du cerne $t-1$ sur le cerne $t$ est très réduit.
Il est bien établi qu'un des effets immédiats de la contrainte hydrique pour le végétal est le ralentissement de sa croissance qui intervient avant tout changement notable de la photosynthèse. À un degré de criticité plus élevé apparaissent des phénomènes de dysfonctionnement hydraulique (cavitation et embolie) et des déficiences de réserves carbonées (Delaporte, 2015). Dans ces conditions, le caractère anisohydrique du cèdre de l'Atlas ne peut être qu'un paramètre aggravant la déshydratation et in fine le dépérissement (Zine El Abidine et al., 2013).

Malgré leur relative proximité $(60 \mathrm{~km})$, les deux massifs d'étude présentent des différences dans leurs réponses à la variabilité climatique. Par son altitude prononcée, ses sols évolués et profonds de type rendzine et sa structure jardinée, le Chélia semble être relativement protégé des événements secs, sévères, longs et fréquents qu'a connus la région des Aurès. Au contraire, les populations équiennes du Belezma, avec leur âge avancé (perte de vigueur) et évoluant à des altitudes plus basses sur des sols squelettiques (lithosols), présentent des conditions plus vulnérables au phénomène de dépérissement. Dans ce contexte, Aoubouazza (2017) affirme que les rendzines, les andosols et les dolomies forment de vrais réservoirs aquifères et atténuent considérablement le phénomène de dépérissement au sein des formations à cèdre de l'Atlas. Le Houerou et al. (1977) constatent que sur nos sites d'étude il existe une différence importante de pluviosité entre versants exposés aux pluies et ceux en ombre pluviale. Dans ce sens, Abdessemed (1981) confirme, également, l'existence d'un déterminisme basé sur le substrat et l'exposition qui différencie les cédraies des Aurès. Meharzi (1994) constate dans le massif de l'Aurès un contraste pluviométrique très prononcé. Il note que le total des précipitations d'une station à une autre peut varier de un à quatre, et même plus sur une distance très courte. L'auteur ajoute que la répartition des pluies obéit à deux paramètres majeurs que sont l'hypsométrie et la disposition du relief. Suivant ces deux paramètres, il établit une configuration pluviométrique de la région définissant trois secteurs : un secteur humide où se situe le massif du Chélia, un secteur moyennement arrosé et un secteur sec auquel est rattaché le massif du Belezma.

Les nuances distinguant les deux massifs forestiers peuvent être déterminantes à ces latitudes où les capacités adaptatives des espèces sont mises à rude épreuve. Ces zones limites des aires biogéographiques des essences aussi nobles et exigeantes que le cèdre de l'Atlas constituent des biotopes très vulnérables où toute perturbation peut porter un préjudice majeur au maintien de ces espèces. Les dépérissements massifs des cédraies des Aurès induits par ces épisodes xériques en sont très explicites (Kherchouche et al., 2013 ; Slimani, 2014).

Les $A C$ sont plus nombreuses au sein des populations du Belezma (une moyenne de $17 \mathrm{AC}$ ) que de celles du Chélia (une moyenne de $11 \mathrm{AC}$ ). Parmi ces années remarquables, on distingue un effectif de sept AC communes (1976, 1978, 1988, 1996, 1997, 2001 et 2002) aux six populations étudiées (figure 5). Les valeurs SPI correspondant à ces années exceptionnelles à portée locale et régionale (Delwaide et Filion, 2010) expliquent leur singularité : les SPI des années 
1976 et 1996 sont considérés " modérément humides ", ceux des années 1997 et 2001 sont qualifiés de « modérément secs » et ceux des années 1978, 1988 et 2002 sont identifiés comme " très secs ". Kherchouche et al. (2013) identifient l'année 1978 comme l'année la plus sèche qui a précédé l'épisode de dépérissement observé dans les cédraies de l'Aurès. Pour Slimani (2014), l'année 1978 est le second événement le plus sec répertorié après celui de l'année 2002.

Comparativement aux chronologies maîtresses par station, les chronologies globales des deux sites d'étude enregistrent un nombre plus élevé d'AC communes (11) dont une majorité (64\%) représente des années sèches (1967, 1978, 1988, 1994, 1997, 2001 et 2002) et moins d'un tiers est représenté par des années humides (1976, 1990, 1992 et 1996). La série du Belezma est la plus impactée par ces événements critiques (figure 5). En outre, une régression sur les indices de croissance inférieurs à la moyenne a révélé une augmentation significative ( $p<0,001)$ des cernes minces au cours des dernières décennies. D’après Slimani et al. (2014), la sécheresse augmente le synchronisme entre les patrons de croissance, car elle homogénéise les conditions écologiques entre sites. En effet, ces derniers répondent concomitamment à la variabilité des conditions mésoclimatiques (climat régional) aux dépens de la réponse au microclimat. En revanche, les conditions humides, engendrées par les précipitations et l'humidité relative (Aussenac et al., 1981), permettent une meilleure expression des facteurs écologiques locaux et par conséquent un indice de croissance différemment enregistré. Les mêmes événements extrêmes sont signalés dans des études antérieures des cédraies d'Algérie et du Maroc (Kherchouche et al., 2013 ; Slimani, 2014 ; Aoubouazza, 2017 ; Navarro-Cerrillo et al., 2019 ; Sarmoum et al., 2019 ; Moukrim et al., 2020).

La relation cerne-climat, exprimée par les corrélations saisonnières, révèle un plus fort déterminisme des régresseurs climatiques ( $P$ et $T$ ) sur la croissance radiale dans le massif du Belezma (figure 6). En considérant les mois individuellement, les précipitations de l'année $t-1$ des mois de septembre au Chélia et de septembre et octobre au Belezma agissent positivement sur la croissance radiale de l'année $t$. Cependant, cette corrélation est d'autant plus homogène entre les deux sites en considérant les précipitations moyennes sur des périodes plus longues (6 et 12 mois). Une ressource hydrique qui sera vraisemblablement responsable de la reconstitution des réserves en eau du sol et profitable à la reprise de la croissance à la fin de l'hiver. Ces résultats corroborent ceux rapportés dans les travaux de Kherchouche et al. (2013) et Slimani et al. (2014) pour la même région d'étude.

La pluviométrie reçue pendant les mois de janvier, février, mars, avril et mai au Belezma et durant le mois de mars au Chélia reste déterminante pour la mise en place de l'accroissement annuel. L'expression de la précipitation en tant que facteur limitant est plus importante au Belezma. En revanche, conjuguées au déficit hydrique, les hautes températures de juin affectent négativement et significativement la croissance radiale au Belezma, pouvant induire un ralentissement voire un arrêt précoce de la croissance radiale. Elles n'ont, cependant, aucune incidence pour le Chélia qui semble être privilégié par ses caractéristiques mésologiques et la structure jardinée de ses peuplements. Ce résultat s'accorde avec ceux de Derak et al. (2008) qui soulignent que les cédraies saines au Maroc sont associées aux structures irrégulières et jardinées.

À l'image des peuplements de pin noir en région méditerranéenne étudiés par Sangüesa-Barreda et al. (2019), la cédraie du Belezma a montré une augmentation conséquente des corrélations cernes-précipitations mensuelles à partir de la seconde moitié du XXe siècle. Cela se justifie par la tendance à la baisse de l'indice SPI et la récurrence accrue des épisodes de sécheresse au cours de cette période. Cette situation est aggravée par l'accroissement des températures moyennes annuelles depuis la fin des années 1970 (figure 3) (Farah, 2014).

\section{Conclusion}

Les résultats de cette étude basée sur une approche dendrochronologique ont montré une croissance des arbres très instable à partir de la seconde moitié du XXe siècle. L'augmentation des événements critiques au cours des dernières décennies a engendré une forte variabilité de l'indice de croissance au sein de ces formations aurésiènnes à cèdre de l'Atlas. Le degré de stress hydrique enregistré, reflété par l'augmentation significative des années caractéristiques, a permis un meilleur synchronisme des patrons de la croissance radiale et une réponse accrue des arbres au signal climatique perçu. Le déterminisme des précipitations printanières est un élément clé dans la formation du cerne annuel de croissance. Néanmoins, les précipitations automnales et hivernales jouent un rôle prépondérant dans la survie du cèdre ; elles améliorent les réserves utiles en eau du sol que le cèdre de l'Atlas utilise pendant la phase d'activité cambiale.

Les sécheresses fréquentes et l'augmentation des températures au niveau de ces limites méridionales de l'aire biogéographique du cèdre l'Atlas ont fait dériver ces cédraies vulnérables vers des points de ruptures (dépassement de la limite de résilience où le dépérissement devient inévitable) parfois irréversibles. Certains facteurs mésologiques tels qu'une altitude élevée, une exposition fraîche, un sol profond, une faible densité et une structure jardinée des peuplements (arbres de tous âges et toutes dimensions en mélange intime) s'avèrent déterminants dans l'atténuation et la compensation de l'effet stress hydrique et par conséquent du degré de dépérissement.

Le stress hydrique survenu au sein de ces formations peut être considéré comme un facteur déclenchant du phénomène de dépérissement, qui est parfois aggravé par les facteurs propres à chaque peuplement. Concernant l'effet de la densité des peuplements, il est important de préciser que dans cette étude ce paramètre n'a pas été considéré comme une variable statistique explicative de la variabilité de croissance radiale mais uniquement comme grandeur descriptive pouvant être considérée comme un des éléments de réponse à cette variabilité. 
Dans le souci de la conservation du cèdre de l'Atlas, la mise en place d'opérations sylvicoles dans les cédraies dépéries est une nécessité pour réduire les contraintes de gestion de l'eau utile. Malgré les contraintes imposées par le Parc national du Belezma, qui interdisent l'exploitation des arbres, une gestion rationnelle des peuplements par des prélèvements sélectifs des arbres ne peut que contribuer à réduire les compétitions très accrues observées dans les peuplements et permettre une meilleure alimentation des réserves hydriques. De plus, dans certaines conditions, l'ouverture des peuplements âgés et fermés, par des opérations sylvicoles appropriées, facilitera la régénération naturelle et permettra l'installation d'une structure irrégulière très recommandée dans ces cédraies perturbées.

\section{Remerciements}

Nos remerciements s'adressent à M. Frédéric Guibal pour son chaleureux accueil au sein du laboratoire de dendrochronologie de l'Institut méditerranéen de biodiversité et d'écologie marine et continentale, Aix-en-Provence, France. Nous remercions également les personnels de la circonscription des forêts de Bouhmama (Khenchela) et du Parc national du Belezma (Batna) pour leurs aides précieuses accordées durant la réalisation de ce travail. Un hommage particulier est rendu, ici, à feu Dr Mahand Messaoudène, un des pionniers de la dendrochronologie algérienne, initiateur de ce travail de recherche.

\section{Références}

Abdessemed K., 1981. Le cèdre de l'Atlas (Cedrus atlantica Manetti) dans les massifs de l'Aurès et du Belezma. Étude phytosociologique, problèmes de conservation et d'aménagement. Thèse de docteur ingénieur, Université d'Aix-Marseille III, France, 119 p. https://bibliotheques. mnhn.fr/medias/detailstatic.aspx?INSTANCE=EXPLOITATION\&RSC BASE $=$ HORIZON\&RSC DOCID $=468576$

Allen C. D., Macalady A. K., Chenchouni H., Bachelet D., McDowell N., Vennetier M., et al., 2010. A global overview of drought and heat-induced tree mortality reveals emerging climate change risks for forests. Forest Ecology and Management, 259 (4): 660-684. https://doi.org/10.1016/j.foreco.2009.09.001

Alileche A., 2012. Étude du dépérissement du cèdre de l'Atlas (Cerdrus altantica Manetti) dans la cédraie des Aurès. Mémoire magistère, Université des sciences et de la technolgie Houari Boumédiène, Alger, Algérie, 90 p. https://repository.usthb.dz/ handle/123456789/2921

Aoubouazza M., 2017. Influence des propriétés hydrodynamiques du sol sur la sensibilité au dépérissement du Cèdre dans le Causse moyenatlasique central marocain. Revue Marocaine des Sciences Agronomiques et Vétérinaires, 5 (3) : 293-303. https://www.agrimaroc.org/index.php/ Actes_IAVH2/article/view/505

Aussenac G., Granier A., Gross P., 1981. Étude de la croissance en hauteur du Cèdre (Cedrus atlantica Manetti). Utilisation d'un appareillage de mesure automatique. Annales des Sciences Forestières, 38 (3) : 301316. https://doi.org/10.1051/forest:19810301

Bentouati A., 2008. La situation du cèdre de l'Atlas en Algérie. Forêt Méditerranéenne, 29 (2) : 203-208. http://www.foret-mediterraneenne. org/fr/catalogue/id-1108-la-situation-du-cedre-de-l-atlas-en-algerie

Cuccia C., 2013. Impacts du changement climatique sur la phénologie du Pinot noir en Bourgogne. Thèse de doctorat en géographie, Université de Bourgogne, France, 321 p. https://www.theses.fr/2013DIJ0S094
Cook E. R., 1985. A time series analysis approach to tree ring standardization. PhD dissertation, University of Arizona, Department of Geosciences, USA, 36 p. https://www.st-andrews.ac.uk/ rjsw/ PalaeoPDFs/Cook1985-Chapter\%202.pdf

Cook E. R., Briffa K. R., Meko D. M., Graybill D. A., Funkhouser G., 1995. The 'segment length curse' in long tree-ring chronology development for palaeoclimatic studies. The Holocene, 5 (2): 229-237. https://doi. org/10.1177/095968369500500211

Delaporte A., 2015. Vers une compréhension fonctionnelle des dépérissements forestiers : étude du cas du hêtre (Fagus sylvatica L.) en forêt de Fontainebleau. Thèse de doctorat, Université Paris-Sud, France, 150 p. https://tel.archives-ouvertes.fr/tel-01216464/document

Delwaide A., Filion L., 2010. Échantillonnage et datation dendrochronologique. In : Filion L., Payette S. (éds). La dendroécologie : Principes, méthodesetapplications. Québec,Canada,Pressesdel'Université Laval, 167-197. https://www.researchgate.net/publication/285772202 Echantillonnage et datation dendrochronologique

Demarteau M., François L., Cheddadi R., Roche E., 2007. Réponses de Cedrus atlantica aux changements climatiques passés et futurs. Revue Internationale de Géologie, de Géographie et d'Écologie Tropicales, 31 : 105-146. http://hdl.handle.net/2268/115919

Derak M., M'hirit O., Mouflih B., Et-Tobi M., 2008. Influence de la densité et du type de peuplement sur le dépérissement du Cèdre à Sidi M'Guild (Moyen Atlas marocain). Forêt Méditerranéenne, 29 (1) : 23-32. http://hdl. handle.net/2042/39042

Farah A. K., 2014. Changement climatique ou variabilité climatique dans l'Est algérien. Mémoire de magistère, Université de Constantine, Algérie, 127 p. http://www.secheresse.info/spip.php?article80242

Fritts H., 1976. Tree rings and climate. London, United Kingdom, Academic Press, 534 p. https://www.elsevier.com/books/tree-rings-and-climate/ fritts/978-0-12-268450-0

Harfouche A., Nedjahi A., 2003. Prospections écologiques et sylvicoles dans les cédraies du Belezma et de l'Aurès à la recherche de peuplements semenciers et d'arbres plus. Revue Forestière Française, 55 (2) : 113-122. https://doi.org/10.4267/2042/5162

Harris I., Jones P. D., Osborn T. J., Lister D. H., 2014. Updated highresolution grids of monthly climatic observations - the CRU TS3.10 Dataset. International Journal of Climatology, 34: 623-642. https://doi. org/10.1002/joc.3711

Hirche A., Boughani A., Salamani M., 2007. Évolution de la pluviosité annuelle dans quelques stations arides algériennes. Science et changements planétaires / Sécheresse, 18 (4) : 314-320. https://www.jle.com/10.1684/ sec.2007.0099

Holmes R. L., 1983. Computer-assisted quality control in tree-ring dating and measurement. Treering Bulletin, 43: 51-67. https://www. Itrr.arizona. edu/ ellisqm/outgoing/dendroecology2014/readings/Holmes 1983.pdf

Jacq V., 2008. Les modèles de prévision climatique en région méditerranéenne. Forêt Méditerranéenne, 29 (2) : 107-112. http://pascalfrancis.inist.fr/vibad/index.php?action=getRecordDetail\&idt=20657343

Kherchouche D., Kalla M., Gutierrez E., Briki A., Hamchi A., 2013. La sécheresse et le dépérissement du cèdre de l'Atlas (Cedrus atlantica Manetti) dans le massif du Belezma (Algérie). Science et changements planétaires / Sécheresse, 24 (2) : 129-137. http://doi.org/10.1684/ sec.2013.0384

Lapie G., 1909. Étude phytogéographique de la Kabylie du Djurjura. Thèse de doctorat, Université de Paris, France, 156 p. https://ia600306. us.archive.org/2/items/etudephytogog00lapi/etudephytogog00lapi.pdf

Le Houerou H., Claudin J., Pouget J., 1977. Étude bioclimatique des steppes algériennes. Bulletin de la Société d'Histoire Naturelle de l'Afrique du Nord, Alger, 68 (34) : 33-74. http://horizon.documentation.ird.fr/exldoc/pleins textes/divers09-04/29910.pdf

Lebourgeois F., Mérian P., 2012. Principes et méthodes de la dendrochronologie. AgroParisTech, ENGREF, 85 p. https://hal.archivesouvertes.fr/cel-01627048

M'hirit O., Benzyane M., 2006. Le Cèdre de l'Atlas : Mémoire du temps. Sprimont, Belgique, Mardaga, Casablanca, Maroc, La croisée des chemins, 288 p. https://books.google.dz/ books?id $=6 \mathrm{WFPkWJOPTEC} \& \mathrm{pg}=$ PA3 $\& \mathrm{hl}=$ fr $\&$ source $=$ gbs selected pages $\&$ cad $=3 \# \mathrm{v}=$ onepage $\& q \& \mathrm{f}=\mathrm{false}$ 
McKee T. B., Doesken N. J., Kleist J., 1993. The relationship of drought frequency and duration to time scales. Proceedings of the 8th Conference on Applied Climatology. Boston, USA, American Meteorological Society, 17, 179-183. https://climate.colostate.edu/pdfs/relationshipofdroughtfrequency.pdf

Meharzi M. K., 1994. Le rôle de l'orographie dans la répartition spatiale des précipitations dans le massif de l'Aurès. Méditerranée, 3 (4) : 73-78. https://www.persee.fr/docmedit_0025-8296_1994_num_80_3_2861

Meko D., Touchan R., Anchukaitis K., 2011. Seascorr: A MATLAB program for identifying the seasonal climate signal in an annual tree-ring time series. Computers \& Geosciences, 37 (9): 1234-1241. https://doi. org/10.1016/i.cageo.2011.01.013

Messaoudène M., Rabhi K., Megdoud A., Sarmoum M., DahmaniMegrerouche M., 2013. État des lieux et perspectives des cédraies algériennes. Forêt Méditerranéenne, 34 (4) : 1-8. http://hdl.handle. net/2042/53617

Moukrim S., Lahssini S., Rifai N., Menzou K., Mharzi-Alaoui H., Labbaci A., et al., 2020. Modélisation de la distribution potentielle de Cedrus atlantica Manetti au Maroc et impacts du changement climatique. Bois et Forêts des Tropiques, 344 (2) : 3-16. https://doi.org/10.19182/ bft2020.344.a31888

Navarro-Cerrillo R. M., Sarmoum M., Gazol A., Abdoun F., Camarero J. J., 2019. The decline of Algerian Cedrus atlantica forests is driven by a climate shift towards drier conditions. Dendrochronologia, 55: 60-70. https:// doi.org/10.1016/j.dendro.2019.04.003

Neuwirth B., Schweingruber F. H., Winiger M., 2007. Spatial patterns of central European pointer years from 1901 to 1971 . Dendrochronologia, 24 (2-3): 79-89. https://doi.org/10.1016/i.dendro.2006.05.004

Nouaceur Z., Laignel B., 2015. Caractérisation des évènements pluviométriques extrêmes sur la rive Sud du bassin méditerranéen : études du cas du « quart Nord-Est algérien ». Actes du XXVIII" Colloque de l'Association internationale de climatologie, Liège, 573-578. http://www. climato.be/aic/colloques/actes/ACTES AIC2015/5\%20Variabilites\%20 et\%20aleas\%20climatiques/093-NOUACEUR-573-578.pdf

Quézel P., 1998. Cèdres et cédraies du pourtour méditerranéen : signification bioclimatique et phytogéographique. Forêt Méditerranéenne, 19 (3) : 243-260. http://www.foret-mediterraneenne.org/fr/catalogue/ id-721-cedres-et-cedraies-du-pourtour-mediterraneen-significationbioclimatique-et-phytogeographique

Quézel P., Médail F., 2003. Écologie et biogéographie des forêts du bassin méditerranéen. Paris, France, Elsevier, 575 p. https://www.lavoisier.fr/ livre/environnement/ecologie-et-biogeographie-des-forets-du-bassinmediterraneen/quezel/descriptif-9782842994518

Sangüesa-Barreda G., Camarero J. J., Sánchez-Salguero R., Gutiérrez E., Linares J. C., Génova M., et al., 2019. Droughts and climate warming desynchronize Black pine growth across the Mediterranean Basin. Science of The Total Environment, 697, 13 p. https://doi.org/10.1016/i. scitotenv.2019.133989

Sarmoum M., Navarro-Cerrilo R., Guibal F., 2019. Bilan actuel et rétrospectif du dépérissement du cèdre de l'Atlas dans le Parc national de Theniet $E \mathrm{E}$ Had (Algérie). Bois et Forêts des Tropiques, 342 (4) : 29-40. https://doi. org/10.19182/bft2019.342.a31636

Sbabdji M., El Hadi O., Haddad A., Kadik B., Lambs L., 2009. Cedar tree growth (Cedrus atlantica Manetti) in Chréa National Park, Algeria, and the influence of defoliation by the pine processionary caterpillar (Thaumetopoea pityocampa Schiff.). Revue d'Écologie (La Terre et la Vie), 64 : 323-332. http://hdl.handle.net/2042/55794

Sbabdji M., Lambs L., Haddad A., Kadik B., 2015. Effect of periodic defoliations by Thaumetopoea pityocampa Schiff. on radial growth in cedar woodland in Chréa, Algeria. Revue d'Écologie (La Terre et la Vie), 70: 371-386. http://hdl.handle.net/2042/56930

Schweingruber F. H., Eckstein D., Serre-Bachet F., Bräker O. U., 1990. Identification, presentation and interpretation of event years and pointer years in dendrochronology. Dendrochronologia, 8: 9-38. https://www. naturfagsenteret.no/c1761334/binfil/download2.php?tid =1856970

Slimani S., 2014. Reconstitutions dendrochronologiques du climat et de l'historique des incendies dans les régions des Aurès et de Kabylie, nord de l'Algérie. Thèse de doctorat, Université Mouloud Mammeri, Tizi-Ouzou, Algérie, 171 p. https://vrelex.ummto.dz/bitstream/handle/ummto/1575/ Th\%c3\%a8se 5.\%20Slimani 2014.pdf? sequence=1\&isAllowed=y
Slimani S., Derridj A., Gutierrez E., 2014. Ecological response of Cedrus atlantica to climate variability in the Massif of Guetiane (Algeria). Forest Systems, 23 (3): 448-460. http://dx.doi.org/10.5424/fs/2014233$\underline{05175}$

Stokes M. A., Smiley T. L., 1996. An introduction to tree-ring dating. Tucson, AZ, USA, University of Arizona Press, 73 p. https://uapress. arizona.edu/book/an-introduction-to-tree-ring-dating

Touchan R., Anchukaitis K. J., Meko D. M., Attalah S., Baisan C., Aloui A., 2008. Long term context for recent drought in northwestern Africa. Geophysical Research Letters, 35 (13), 5 p. https://agupubs.onlinelibrary. wiley.com/doi/epdf/10.1029/2008GL034264

Touchan R., Anchukaitis K. J., Meko D. M., Kerchouche D., Slimani S., Ilmen R., et al., 2017. Climate controls on tree growth in the Western Mediterranean. The Holocene, 27: 1429-1442. https://doi. org/10.1177/0959683617693901

Véla E., Benhouhou S., 2007. Évaluation d'un nouveau point chaud de biodiversité végétale dans le Bassin méditerranéen (Afrique du Nord). Comptes Rendus Biologies, 330 (8) : 589-605. https://doi.org/10.1016/i. crvi.2007.04.006

Zine El Abidine A., Lamhamedi M. S., Taoufik A., 2013. Relations hydriques des arbres sains et dépérissants de Cedrus atlantica M. au Moyen Atlas Tabulaire au Maroc. Revue Internationale de Géologie, de Géographie et d'Écologie Tropicales, 37 (2) : 157-176. https://mffp. gouv.qc.ca/documents/forets/connaissances/recherche/Geo-EcoTrop-37-2-157-176.pdf

\begin{tabular}{|c|c|}
\hline Rôle du contributeur & Noms des auteurs \\
\hline Conceptualisation & M. Tafer, M. Messaoudène \\
\hline Gestion des données & M. Tafer \\
\hline Analyse formelle & M. Tafer, S. Slimani, D. Kherchouche \\
\hline $\begin{array}{l}\text { Acquisition du } \\
\text { financement }\end{array}$ & M. Tafer \\
\hline $\begin{array}{l}\text { Enquête et } \\
\text { investigation }\end{array}$ & M. Tafer \\
\hline Méthodologie & M. Tafer \\
\hline Gestion de projet & M. Tafer, F. Bekdouche \\
\hline Ressources & M. Tafer, D. Kherchouche \\
\hline Logiciels & M. Tafer, S. Slimani, D. Kherchouche \\
\hline Supervision & M. Tafer, F. Bekdouche \\
\hline Validation & $\begin{array}{l}\text { M. Tafer, F. Bekdouche, S. Slimani, } \\
\text { D. Kherchouche }\end{array}$ \\
\hline Visualisation & $\begin{array}{l}\text { M. Tafer, F. Bekdouche, S. Slimani, } \\
\text { D. Kherchouche }\end{array}$ \\
\hline $\begin{array}{l}\text { Écriture - Préparation } \\
\text { de l'ébauche originale }\end{array}$ & M. Tafer, S. Slimani \\
\hline $\begin{array}{l}\text { Écriture - Révision } \\
\text { et édition }\end{array}$ & $\begin{array}{l}\text { M. Tafer, F. Bekdouche, S. Slimani, } \\
\text { D. Kherchouche }\end{array}$ \\
\hline
\end{tabular}

Bois et Forêts des Tropiques - Revue scientifique du Cirad -

(c) Bois et Forêts des Tropiques (c) Cirad

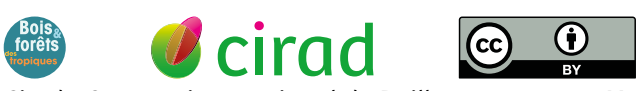

Cirad - Campus international de Baillarguet, 34398 Montpellier Cedex 5, France - Contact : bft@cirad.fr - ISSN : L-0006-579X 